\begin{tabular}{ccc}
\hline & International Journal of Engineering \& Technology, 7(3.33)(2018) 106-108 \\
SPC & Website: www.sciencepubco.com/index.php/IJET & Rechnology \\
\hline
\end{tabular}

\title{
A Mutual Awareness Mechanism of Inter-MVFs (Mobile Virtual Fences) to Support Connected Car Service
}

\author{
Inhwan Kim ${ }^{1}$, Hyunmi Yoo ${ }^{2}$, Eom Young-Hyun ${ }^{3 *}$, Sungguk Cho ${ }^{4 *}$ and Byungkook Jeon ${ }^{5 *}$ \\ ${ }^{1,2,3,5}$ Dept. of Software, Gangneung-Wonju National University, Wonju City, Gangwon Prov., 26403 Korea \\ ${ }^{4}$ Dept. of Multimedia Engineering, Gangneung-Wonju National University, Wonju City, Gangwon Prov., 26403 Korea \\ *Corresponding author E-mail: jeonbk@gwnu.ac.kr
}

\begin{abstract}
In this paper, we present a mutual awareness mechanism among lots of MVFs(Mobile Virtual Fences) to configure Connected cars service in VANETs(Vehicular Ad hoc Networks). If the MVFs, as our previous researches, mounted on each car are recognized and constructed to each other, the service environment of connected car can be configured and simulated. So it is necessary to have a mechanism that the moving MVFs automatically detect according to the speed. The proposed mutual awareness mechanism is implemented by exchanging messages among inter-MVFs based on context-aware computing. As a result, when the MVFs are mutually recognized, they are basically simulated in a connected car service. Therefore, in the future, the proposed mechanism will be contribute to improve the reliability, safety, and efficiency in the era of autonomous vehicles.
\end{abstract}

Keywords: Mobile Virtual Fence, Mutual Awareness, Connected cars, Vehicle-to-Everything, Vehicular Ad hoc Networks

\section{Introduction}

According to rapid development of the wireless communication technology, VANETs(Vehicular Ad hoc Networks) are dedicated for V2X(Vehicle-to-Everything) communications and extend the communication coverage area by information exchange among vehicles in a distributed[1-5]. As a result, V2X is considered as a promising technology to support safety related applications in CITS(Connected Intelligent Transportation System), which enables moving vehicles to quickly and accurately collect real-time road traffic information and notify neighboring vehicles of potential dangerous accidents rapidly[1,2,3].

Therefore, we propose a mutual awareness mechanism among lots of Mobile Virtual Fences (MVF) instead of connected car on V2X. The MVF as the results of our previous researches is based on the FloGeo and the flexible mobile three-dimensional geofence[6-12]. When the MVFs mounted on each vehicle are recognized and constructed to each other, the service environment of connected car can be configured and simulated. So it is necessary to have a mechanism that the moving MVF s automatically detect according to the speed. The proposed mutual awareness mechanism is implemented by exchanging messages between MVFs based on context-aware computing[13]. As a result, when the MVFs are mutually recognized, they are basically simulated in a service environment of connected car.

The paper begins with a review of background, then goes on to describe a mutual awareness mechanism, before providing experimental results from an early user study, followed by a discussion and conclusion.

\section{Background}

\subsection{Connected Car}

CAMs GM(General Motors) was the first automaker to bring the first connected car features to market with OnStar in 1996, which was created by GM working with Motorola Automotive $[1,14]$. The main purpose was safety and to get emergency help to a vehicle when there was an accident. Nowadays a connected car is a car that is equipped with Internet access, and usually also with a wireless local area network using DSRC(Dedicated Short Range Communication) radios, operating in the FCC-granted $5.9 \mathrm{GHz}$ band with very low latency[1,3,14]. So applications of connected car are mainly single vehicle applications and cooperative safety and efficiency applications.

\subsection{Mobile Virtual Fence}

The MVF is based on the FloGeo as a floatable, flexible threedimensional geofence with mobility[6,7,8]. It is one of geofence models automatically capable of being moved along the movement route of users using location tracking mechanism regardless of whether they are in indoors or outdoors. Also the MVF introduces the concept of a movable three dimensional geofence and describes location analysis mechanism to discover the movement route[6-12]. Furthermore, the MVF can not only provide the stationary and fixed geofencing services but also the flexible, dynamic and movable geofencing service to communicate the intergeofences. 


\section{A Mutual Awareness Mechanism}

For connected car service on V2X, we design of a mutual awareness mechanism inter-MVFs to construct a service environment of connected car. The proposed mechanism recognizes whether the vehicle and the vehicle are connected to each other through the client corresponding to the vehicle driver's mobile device(smartphone, tablet, etc.). The server also analyzes the received message and sends it to the clients.

\subsection{Design of a Client Model}

The client means a mobile device carried by the driver of the vehicle. The operation process includes activating the connected car service and collecting sensor data of the mobile device while the vehicle is moving and transmitting the sensor data to the server. Fig. 1 shows the process of activating the connected car service.

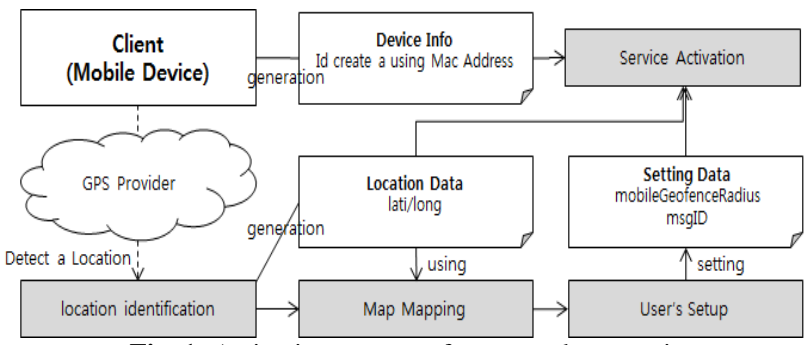

Fig. 1: Activation process of connected car service

In Fig. 1, when the client application is activated, a unique ID of the device is generated based on the Mac address of the mobile device. In addition, the latitude and longitude of the mobile device is determined by the GPS provider, and then the connected car service is activated by setting the MVF radius and the message type by the client.

\subsection{Design of a Server Model}

The server receives the message sent by the client periodically, manages the field of the received message, and analyzes the message. In addition, all the clients' events are handled to configure the connected car service environment.

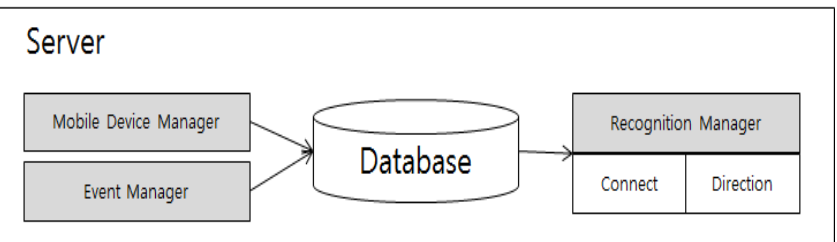

Fig 2: Architecture of the proposed server model

The description of each manager in Fig. 2 is as follows.

- Mobile Device Manager: It manages the client connection and manages the messages sent by each client.

- Event Manager: It manages events generated by clients. In this paper, we manage event about emergency situation.

- Recognition Manager: It analyzes the message sent by the client and manages the connection between the vehicle and the vehicle.

The role of configuring the connected car environment in the server is done by the recognition manager. A method of determining whether a vehicle is connected to a vehicle by analyzing a message sent from each client is performed in two steps. The first step determines if the vehicles are traveling in the same direction in the same area. The second step is to determine the overlap of MVF in vehicles traveling in the same direction in the same direction.

\section{Experimental Results}

In this chapter, we implement the client and server that construct the connected car environment using a mutual awareness mechanism. The client is implemented using a smartphone based on the Android operating system as a mobile device. For this purpose, there are six mobile devices used in the experiment. The same model is numbered in the \# symbol.

- Samsung Galaxy S7 Edge \#1 : 9d1ec2181794e387

- Samsung Galaxy S7 Edge \#2 : 5df9a94721d24ddd

- Samsung Galaxy J7 : 7d4243cc01281ce2

- Samsung Galaxy S3 \#1 : 9d202dee2773a0f1

- Samsung Galaxy S3 \#2 : $28666666273 b c 42 a$

- Samsung Note8 : 1fe4de746312ae93

When these six mobile devices are distributed at each location on the Google map as shown in Fig. 3, we verify that they are connected to the server and connected to each other. After connecting to the server at the location, the distances to the devices and the connection status are shown and the log data is represented in the file as shown in Fig. 4.

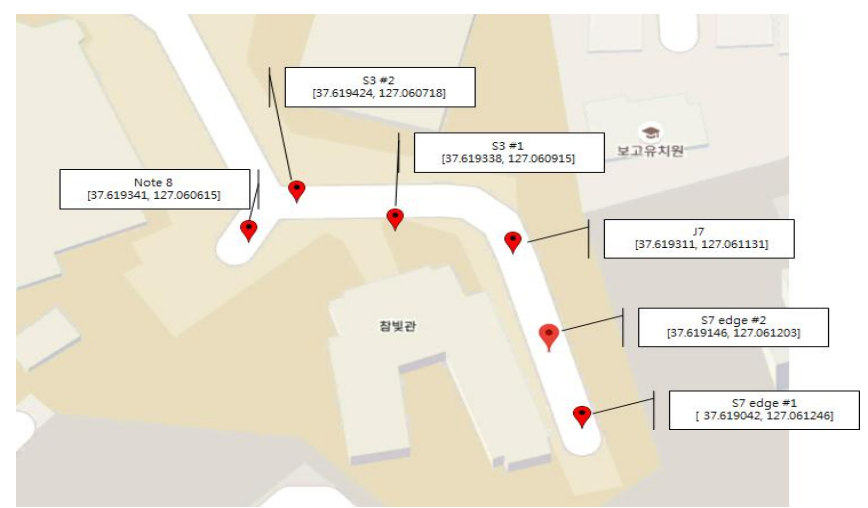

Fig. 3: A screenshot of location applied to all mobile devices on the Google map

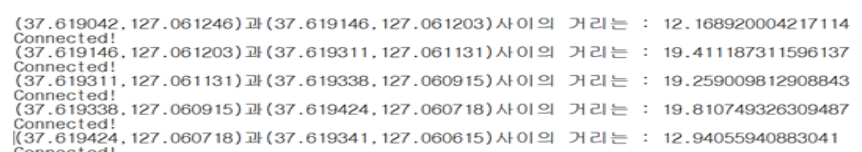

Fig. 4: The $\log$ data of server side

As shown in Fig. 4, the distances of the devices are less than 20 meters, which is the sum of the two radii. Fig. 5 below shows the server UI immediately after all the mobile devices have connected to the server.

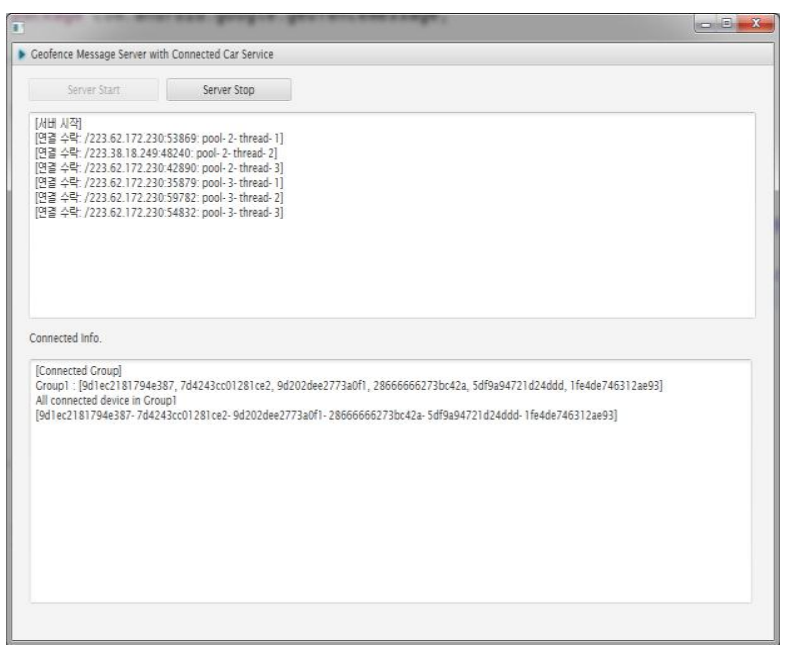

Fig. 5: A screenshot of server side after connection of the clients 
In Fig. 5, the server receives the basic message as soon as it accepts each client's connection and shows that they are concurrently connected to each other. It is confirmed that all six devices are in the same area belonging to the Group 1, and all the devices are connected to each other as a result of 'All connected device in Group 1'.

\section{Conclusion}

In this paper, we designed and implemented a mutual awareness mechanism inter-MVF to configure a service environment of connected car. When the MVFs mounted on each vehicle are sensed and configured to each other, the service environment of connected car can be constructed and simulated. So, the proposed mutual awareness mechanism is implemented by exchanging messages between MVFs based on context-aware computing. As a result, when the MVFs are mutually recognized, they are basically simulated in a connected car environment.

To date, lots of autonomous vehicles have been under development, and some have been equipped with partially autonomous vehicles. In addition, in order to ensure the safety of autonomous vehicles, it is necessary to construct a communicated car environment so that vehicles can communicate with each other. Therefore, in the future, the proposed sensing mechanism will be contribute to improve the reliability, safety, and efficiency in the era of autonomous vehicles.

\section{Acknowledgement}

This research was supported by a grant(18CTAP-C133299-02) from Technology Advancement Research Program funded by Ministry of Land, Infrastructure and Transport of Korean government. Also, this research was supported by Basic Science Research Program through the National Research Foundation of Korea(NRF) funded by the Ministry of Education(NRF2017R1A6A3A01005949), and supported by the Human Resource Training Program for Regional Innovation and Creativity through the Ministry of Education and National Research Foundation of Korea (NRF-2015H1C1A1035548).

\section{References}

[1] John Greenough, The CONNECTED CAR REPORT: Forecasts, technologies, and leading manufacturers, BI Intelligence, Jan., (2016)

[2] Keyvan Golestan, Ridha Soua, Fakhri Karray, Mohamed S. Kamel Situation awareness within the context of connected cars : A comprehensive review and recent trends, Information Fusion 29, (2016), 68-83

[3] Gongjun Yan, Danda B. Rawat, Vehicle-to-vehicle connectivity analysis for vehicular ad-hoc networks, Ad Hoc Networks 58, (2017), 25-35

[4] F. Dressler et al., Inter-Vehicle Communication - Quo Vadis, IEEE Communications Magazine, 52(6), (2014) 170-177

[5] G. Karagiannis et al., Vehicular Networking: a Survey and Tutorial on Requirements, Architectures, Challenges, Standards and Solutions, IEEE Communications Surveys and Tutorials, 13(4), pp. 584-616, 2011.

[6] Young-Keun Choi, Sungkuk Cho, Sungkon Park, Young-Hyun Eom, Inhwan Kim, Byungkook Jeon, "An extended threedimensional Geofence platform with rule-based contextawareness service for the internet of things", Journal of Engineering Technology, Vol. 6(1), Jan., (2018), 318-828.

[7] Eom Young-Hyun, Choi Young-Keun, Sungkuk Cho and Byungkook Jeon, FloGeo: A Floatable Three-Dimensional Geofence with Mobility for the Internet of Things, Journal of Advanced Research in Dynamical and Control Systems, Vol. 8, Aug., (2017), 114-120

[8] Eom Young-Hyun, Choi Young-Keun, Sungkuk Cho and Byungkook Jeon A Flexible Mobile-Geofence to support Connected-Cars Technology, Journal of SmartMedia, Sep., (2017), 89-94
[9] Eom Young-Hyun, Choi Young-Keun, Sungkuk Cho and Byungkook Jeon, TemG : A Geofence Platform with TimeLimited Property, The Journal of The Institute of Internet, Broadcasting and Communication (IIBC), Vol. 16, Feb., (2016), 177 182

[10] Eom Young-Hyun, Choi Young-Keun, Sungkuk Cho and Byungkook Jeon, A Mechanism to identify Indoor or Outdoor Location for Three Dimensional Geofence, The Journal of The Institute of Internet, Broadcasting and Communication (IIBC), Vol. 16, 2016, Feb., pp.169-175

[11] Byungkook Jeon, Sungkuk Cho, Design of 3D Geofence Model by Location-aware Mechanism, INFORMATION, Vol. 18, 2015, July, pp. 3175-3180

[12] Eom Young-Hyun, Choi Young-Keun, Sungkuk Cho and Byungkook Jeon, Design and Implementation of a Framework of Three-Dimensional Geofence, INFORMATION, Vol. 19, 2016 , Sep., pp. 3895-3900.

[13] Charith Perera, Arkady Zaslavsky, Peter Christen, Dimitrios Georgakopoulos, Context Aware Computing for The Internet of Things: A Survey, IEEE Comm. Surveys Tutorials, 2013, pp.1-44

[14] Wikipedia, https://en.wikipedia.org/wiki/Connected_car 